\title{
Faible différenciation génétique, à partir d'amplification aléatoire d'ADN polymorphe (RAPD), entre les types de pin sylvestre (Pinus sylvestris L.) d'altitude et de plaine dans les Alpes à climat continental
}

\author{
Nicolas FourniER $^{\mathrm{a}, \mathrm{b}}$, Andreas RIGLING $^{\mathrm{a}}$, Matthias DobBERTIN $^{\mathrm{a}}$, Felix GUGERLI $^{\mathrm{a} *}$ \\ a Institut Fédéral de Recherches WSL, Zürcherstrasse 111, 8903 Birmensdorf, Suisse \\ ${ }^{\mathrm{b}}$ Chaire de Sylviculture, École Polytechnique Fédérale de Zürich, Rämistrasse 101, 8092 Zürich, Suisse
}

(Reçu le 4 mars 2005 ; accepté le 7 septembre 2005)

\begin{abstract}
Résumé - En Valais (Suisse), deux types de pin sylvestre (Pinus sylvestris L.) se distinguent morphologiquement et écologiquement : un pin de plaine et un pin d'altitude. Les pinèdes de plaine présentent des signes de dépérissement depuis le début des années 1990. Quatre paires de populations ont été choisies dans cette vallée et deux autres dans deux autres régions alpines à climat continental. La différenciation génétique entre les deux types et entre deux classes de vitalité a été étudiée à l'aide d'amplification aléatoire d'ADN polymorphe (RAPD). La croissance en diamètre et la quantité d'aubier des deux types ont été mesurées. À l'exception d'une paire de populations, les deux types se distinguent génétiquement, mais faiblement $\left(\Phi_{\mathrm{ST}}=4,2 \%\right.$ à 5,8\%). Une sélection différenciée avec l'altitude, la recolonisation à partir de différents refuges glaciaires, un isolement phénologique et les vents dominants sont les facteurs de différenciation envisagés. Aucune raison génétique au dépérissement n'a été trouvée. Les différences de croissance et dans la quantité d'aubier soutiennent une adaptation aux régimes hydriques locaux.
\end{abstract}

écotypes / RAPD / cernes de croissance / dépérissement forestier / variation génétique

\begin{abstract}
Random amplified polymorphic DNA (RAPD) patterns show weak genetic differentiation between low- and high-elevation types of Scots pine (Pinus sylvestris L.) in dry continental valleys in the Alps. Two types of Scots pine (Pinus sylvestris L.), found either in the lowland or in the subalpine zone, are morphologically distinguished in the central-Alpine Valais (Switzerland), a dry continental valley. The low-elevation pine forests show signs of die-back since the early 1990s. We selected four elevational population pairs in the area, and two additional pairs in two other central-Alpine regions. Genetic differentiation between the two pine types, i.e. between elevations, and between two classes of vitality were studied using random amplified polymorphic DNA (RAPD). Growth in diameter and sapwood proportion was further assessed. Population pairs, with one exception, showed weak, but significant genetic differentiation $\left(\Phi_{\mathrm{ST}}=4.2 \%-5.8 \%\right)$. On the other hand, there was no correlation between genetic data and the die-back observed. We consider disruptive evolution, postglacial migration, phenological separation or wind directions as possible explanations. Differences in growth and sapwood proportions supported the assumption of adaptation to local hydrological regimes.
\end{abstract}

ecotypes / RAPD / growth rings / forest die-back / genetic variation

\section{INTRODUCTION}

Le pin sylvestre présente une large variabilité phénotypique à travers son aire d'expansion. De nombreuses tentatives destinées à établir un système taxonomique uniforme et cohérent ont été réalisées. Sur la base de critères anatomiques et biochimiques, plus de vingt races et d'innombrables écotypes, formes ou variétés ont été décrits à ce jour [36]. Pour le canton du Valais (Suisse), deux types de pin sylvestre se distinguent morphologiquement, physiologiquement et écologiquement, à savoir un pin de plaine et un pin d'altitude appelés respectivement « pin gris » et «pin rouge », noms donnés localement en raison de la teinte des écorces [21]. Sur les stations sèches de la plaine du Rhône, le pin sylvestre atteint rarement 15 m, possède un faible accroissement radial et une faible proportion de bois de cœur. En altitude, il dépasse fréquemment $25 \mathrm{~m}$ et son accroissement peut atteindre le double de celui du pin sylvestre

\footnotetext{
* Auteur pour correspondance : gugerli@wsl.ch
}

de plaine. Hess [21] considère la forme d'altitude et celle de plaine comme deux écotypes. Il observe par ailleurs une tendance à une différenciation semblable dans les autres vallées alpines à climat continental.

Une part de la diversité du pin sylvestre provient de la répartition des différents refuges glaciaires. Les analyses palynologiques supposent l'existence de refuges dans les Balkans, les Apennins, les Carpates, le sud de la Pologne et la péninsule Ibérique $[1,23]$. Des analyses d'ADN mitochondriaux (restriction fragment length polymorphism, PCR-RFLP) ont apporté certaines précisions sur l'histoire de la recolonisation postglaciaire et l'emplacement des différents refuges de pin sylvestre [51,53]. Les populations situées au Nord de l'arc alpin se distinguent génétiquement de celles du Sud des Alpes [51]. La même observation a été faite à partir de l'analyse chromatographique des monoterpènes [57]. Cette structure laisse envisager des routes distinctes de recolonisation pour le nord et le sud des Alpes, lesquelles pourraient s'être mélangées à l'intérieur du massif. 
Au cours des dernières décennies, de nombreux articles ont traité de la variation génétique adaptative ou neutre des populations de pin sylvestre pour diverses régions et à différentes échelles spatiales [e.g. 12, 15, 18, 26]. Dans les régions qui étaient recouvertes par les glaciers durant la dernière glaciation, les populations présentent généralement l'essentiel de leur variation génétique située à l'intérieur des populations et une faible différenciation entre les populations avec des valeurs $\mathrm{F}_{\mathrm{ST}}$ de l'ordre de $2 \%$ à $3 \%$. En présence d'un gradient écologique abrupt et d'un isolement phénologique, une différenciation génétique à partir d'isozymes a cependant été observée sur de très courtes distances, par exemple entre des pinèdes de marais et de milieux xériques [47]. De même, dans l'Idaho (USA), une différence d'altitude de $300 \mathrm{~m}$ a laissé apparaître des différences génétiques lors d'essais de provenance [31]. À l'aide d'isozymes, Sannikov et al. [48] ont finalement mesuré une distance génétique de $\mathrm{d}_{\mathrm{Nei}}=0,021$ entre des populations distantes de seulement $30 \mathrm{~km}$ mais présentant une différence d'altitude de $500 \mathrm{~m}$, dans les Carpates.

Les différences de croissance et de duraminisation entre le pin gris et le pin rouge du Valais suggérées par Hess [21] peuvent provenir d'une adaptation phénotypique ou génétique au régime hydrique. L'approvisionnement en eau est un facteur majeur de la croissance des plantes, l'intensité de la photosynthèse étant couplée à la transpiration stomatale [25]. La surface de l'aubier détermine la capacité de conduction de la tige, et doit être suffisante pour compenser la perte d'eau des feuilles par la transpiration [50]. Cette relation se manifeste par la corrélation entre la surface de l'aubier (sapwood area, SA) et la surface foliaire (leaf area, LA), pour un arbre individuel [60]. Le modèle hydraulique [62] affirme cependant que le rapport LA/SA dépend de la perméabilité de l'aubier et du climat local. Pour une même surface foliaire, les individus croissant dans des milieux xériques possèdent en effet une surface d'aubier plus élevée [29, 34].

À partir du début des années quatre-vingt-dix, des signes de dépérissement ont été observés à plusieurs reprises sur des parties entières des pinèdes de la région de Viège, dans la plaine du Valais [43]. La majorité des arbres morts présentent une répartition diffuse dont la cause n'est pas évidente. Il semblerait que le processus de dépérissement soit induit par l'action complexe de plusieurs facteurs de stress [43]. Les périodes de sécheresse particulièrement intenses des dernières décennies seraient le facteur déclenchant [10, 11, 42-44]. Ce type de dépérissement des pins ne se limite pas au Valais puisque d'autres régions alpines présentent des symptômes semblables, par exemple en Autriche ou au Val d'Aoste [6, $58]$. Ce modèle étonnant de dépérissement diffus éveille l'intérêt de rechercher une explication génétique. Des différences de résistance entre les provenances et entre les individus d'une même population ont en effet été démontrées à plusieurs reprises pour différents agents pathogènes du pin sylvestre [54].

Dans le présent travail, la différenciation génétique entre les deux types et les classes de vitalité a été étudiée. La méthode d'amplification aléatoire d'ADN polymorphe (random amplified polymorphic DNA, RAPD) a été choisie en raison de la commodité de l'exécution, du grand nombre de marqueurs obtenus et de leur sélection au hasard sur le génome entier.

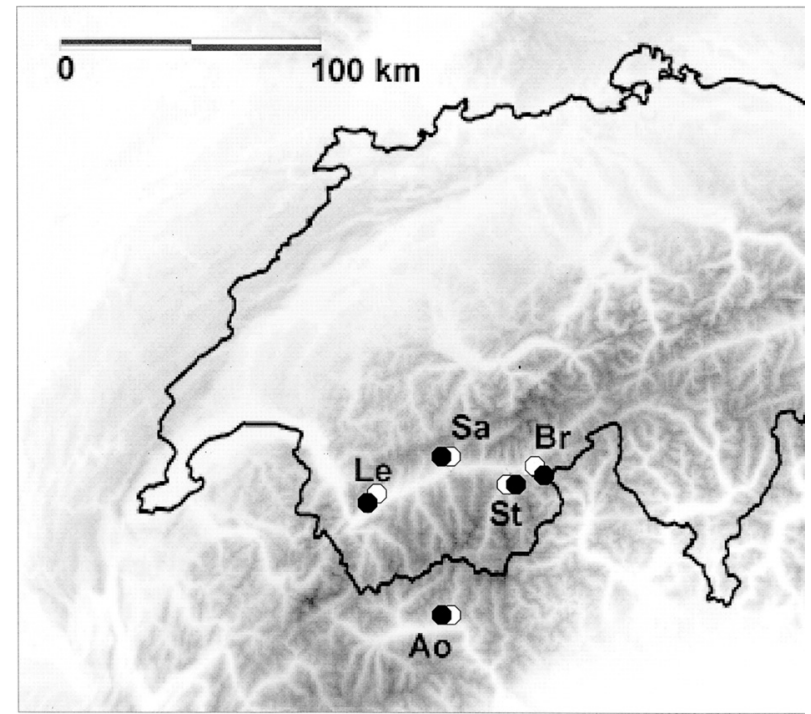

Figure 1. Emplacement des populations étudiées dans le segment ouest de l'arc alpin. La frontière de la Suisse est indiquée. Les points blancs représentent les populations de plaine de Pinus sylvestris et les points noirs celles d'altitude. Les abréviations se réfèrent au tableau I.

Parallèlement à l'analyse génétique, la croissance et le nombre de cernes d'aubier des deux types de plaine et d'altitude ont été mesurés à partir de carottes de bois. Cet article traite des questions suivantes : (i) Les types de plaine et d'altitude peuvent-ils être différenciés à l'aide de marqueurs génétiques? (ii) Existe-t-il, dans le cas du dépérissement du pin, une corrélation entre la vitalité individuelle et des marqueurs génétiques? (iii) Ces deux types présentent-ils des différences de croissance et dans la quantité d'aubier?

\section{MATÉRIELS ET MÉTHODES}

\subsection{Espèce}

Le pin sylvestre (Pinus sylvestris L.) possède en Suisse, la région d'étude principale de ce travail, trois zones de distribution, à savoir les Alpes centrales, le nord-est suisse et le Jura. Son importance est la plus grande dans le Valais où il représente $12 \%$ du nombre de tiges. Le pin sylvestre représente $3,1 \%$ du volume sur pied suisse, ce qui le place au quatrième rang des résineux les plus fréquents [4]. En tant qu'essence pionnière, son amplitude physiologique recouvre tout le domaine de croissance de la forêt. En raison de sa faible compétitivité, il se rencontre principalement sur des stations temporairement hydromorphes ou xéromorphes, sur des sols bien drainés et souvent oligotrophes [59]. Le pin sylvestre est xénogame, son pollen est exceptionnellement bien dispersé par le vent [27]. L'influence humaine a été importante sur la distribution du pin sylvestre en Valais (défrichements, recolonisation naturelle de pâturages abandonnés) mais extrêmement faible du point de vue sylvicole.

\section{2. Échantillonnage}

Six paires de populations ont été choisies sur six transects verticaux. Quatre paires ont été définies en Valais (Suisse), une au Val 
Tableau I. Localisation, abréviation, coordonnées (système suisse), altitude, exposition, hauteur moyenne et vitalité des individus échantillonnés et variance moléculaire des populations étudiées.

\begin{tabular}{|c|c|c|c|c|c|c|c|c|c|}
\hline Région & Paire & Localité & Abréviation & Coordonnées & $\begin{array}{c}\text { Altitude } \\
(\mathrm{m})\end{array}$ & Exposition & $\begin{array}{l}\text { Hauteur } \\
(\mathrm{m})\end{array}$ & $\begin{array}{c}\text { Proportion } \\
\text { forte vitalité ; faible vitalité }\end{array}$ & $\begin{array}{l}\text { Variance } \\
\text { moléculaire }\end{array}$ \\
\hline \multirow[t]{8}{*}{ Valais } & \multirow[t]{2}{*}{ Stalden } & Waldegga & $\overline{\text { St A }}$ & 635’000 / 120’700 & 1700 & $\mathrm{~W}$ & 19 & $13 ; 7$ & 4,42 \\
\hline & & Lärje & St P & $633^{\prime} 850$ / 121'650 & 760 & $\mathrm{~W}$ & 9 & $10 ; 10$ & 3,68 \\
\hline & \multirow[t]{2}{*}{ Brigue } & Obre & $\mathrm{Br} \mathrm{A}$ & $645^{\prime} 150 / 127^{\prime} 350$ & 1450 & S & 18 & $15 ; 5$ & 4,31 \\
\hline & & Bieltini & $\mathrm{Br} \mathrm{P}$ & $641^{\prime} 700$ / 128'200 & 870 & $\mathrm{~N}$ & 9 & $10 ; 10$ & 4,62 \\
\hline & \multirow[t]{2}{*}{ Salquenen } & Plan bois & $\mathrm{Sa} \mathrm{A}$ & $609^{\prime} 600$ / 131'850 & 1680 & S & 17 & $10 ; 10$ & 3,92 \\
\hline & & Groggrü & Sa P & 611'300 / 129'800 & 840 & S & 7 & $10 ; 10$ & 4,29 \\
\hline & \multirow[t]{2}{*}{ Leytron } & Forêt de Sinlio & Le A & $577^{\prime} 850$ / 113'400 & 1420 & SE & 19 & $10 ; 10$ & 4,53 \\
\hline & & Montagnon & Le $\mathrm{P}$ & $581^{\prime} 550 / 115^{\prime} 700$ & 770 & S & 12 & $10 ; 10$ & 4,03 \\
\hline \multirow[t]{2}{*}{ Grisons } & \multirow[t]{2}{*}{ Filisur } & Alvaneu & Fi A & $768^{\prime} 950$ / 172’800 & 1580 & S & 15 & $10 ; 10$ & 3,79 \\
\hline & & Surava & Fi P & $766 ’ 800$ / 170’850 & 980 & $\mathrm{~S}$ & 10 & $10 ; 10$ & 3,86 \\
\hline \multirow[t]{2}{*}{ Aoste } & \multirow[t]{2}{*}{ Aosta } & Cerbio & Ao A & $609^{\prime} 700$ / 070’000 & 1590 & S & 15 & $18 ; 2$ & 4,40 \\
\hline & & Brusoncles & Ao $\mathrm{P}$ & $613^{\prime} 200 / 068^{\prime} 800$ & 940 & SW & 12 & $10 ; 10$ & 3,93 \\
\hline
\end{tabular}

Tableau II. Amorces, séquences $\left(5^{\prime} \rightarrow 3^{\prime}\right)$, concentrations optimales de $\mathrm{MgCl}_{2}$ et d'ADN génomique et amplitude des longueurs de fragments obtenus par PCR de RAPD (d'après Gugerli et al. [19]) de Pinus sylvestris. Le nombre de bandes monomorphes pour l'ensemble des individus est donné entre parenthèses.

\begin{tabular}{|c|c|c|c|c|c|}
\hline \multirow[b]{2}{*}{ Amorce } & \multirow[b]{2}{*}{ Séquence } & \multicolumn{2}{|c|}{ Concentrations pour la PCR } & \multirow{2}{*}{$\begin{array}{l}\text { Amplitude des longueurs } \\
\text { (bp) }\end{array}$} & \multirow{2}{*}{$\begin{array}{c}\text { Nombre de fragments } \\
\text { (monomorphes) }\end{array}$} \\
\hline & & $\mathrm{MgCl}_{2}(\mathrm{mM})$ & $\mathrm{ADN}(\mathrm{ng})$ & & \\
\hline A06 & GGTCCCTGAC & 4,75 & 2,5 & $900-1380$ & $10(1)$ \\
\hline A19 & CAAACGTCGG & 2,75 & 1,0 & $520-1300$ & $12(2)$ \\
\hline B11 & GTAGACCCGT & 4,75 & 2,5 & $400-1100$ & $9(3)$ \\
\hline B12 & CCTTGACGCA & 3,75 & 1,0 & $620-1320$ & $7(2)$ \\
\hline Total & & & & & $38(8)$ \\
\hline
\end{tabular}

d'Aoste (Italie) et une aux Grisons (Suisse), chacune composée d'une population de plaine et d'une population d'altitude (Fig. 1). Le peuplement de plaine a été choisi sur une station aussi sèche que possible. Pour le peuplement d'altitude, la plus grande différence d'altitude a été recherchée (Tab. I). Pour l'analyse génétique, des échantillons d'aiguilles de l'année ont été prélevés sur 20 individus par population. Le degré de vitalité comme indicateur de l'état de santé a été déterminé à partir du degré de transparence de la couronne. Pour ce faire, la défoliation en \% a été évaluée à l'aide du guide photographique des couronnes d'arbres utilisé pour les inventaires Sanasilva en Suisse [55]. Les individus sélectionnés pour l'analyse génétique ont été choisis uniquement dans les deux classes de vitalité extrêmes : «plus de $75 \%$ de perte d'aiguilles » (faible vitalité) et «moins de $25 \%$ de perte d'aiguilles » (forte vitalité). Une distance d'au moins $30 \mathrm{~m}$ entre les individus a été respectée. Une répartition 10:10 a été choisie pour autant que les différences de vitalité aient été suffisantes (Tab. I). Deux carottes de bois par arbre ont été prélevées à hauteur de poitrine, en dehors des zones de bois de réaction.

\subsection{Extraction de l'ADN}

Après avoir été lyophilisés, $50 \mathrm{mg}$ d'aiguilles ont été moulus mécaniquement (MM2000; Retsch). L'extraction de l'ADN génomique a été réalisée à l'aide du DNeasy 96 Plant Kit (QIAGEN) selon le protocole d'extraction du fournisseur, à l'exception du tampon de lavage administré en deux étapes. La qualité d'ADN extrait a été vérifiée sur des gels d'agarose. La quantification a été réalisée par fluorométrie à l'aide du DyNA Quant 200 (Hoefer Pharmacia Biotec).

\subsection{Réaction de polymérisation en chaîne (Polymerase Chain Reaction, PCR)}

Le criblage de 40 amorces de 10-mer (set A et B de Operon Technologies) a permis de sélectionner quatre amorces présentant un électrophorégramme variable et reproductible. Les conditions d'amplification des amorces sélectionnées ont été optimisées pour la quantité d'ADN et pour la concentration de $\mathrm{MgCl}_{2}$ (Tab. II). La concentration des autres réactifs du mélange de PCR d'un volume de $15 \mu \mathrm{L}$ de même que le profil du cycleur thermique (PTC-100, MJ Research) proviennent de Gugerli et al. [19]. Afin de conserver les conditions aussi constantes que possible, un seul mélange réactionnel par amorce a été préparé, à l'exception de la Taq DNA polymérase (Sigma), et congelé en aliquotes jusqu'à l'utilisation. Les produits de PCR ont été séparés par électrophorèse sur des gels d'agarose à $1,5 \%$, colorés dans un bain de bromure d'éthidium et photographiés sous lumière UV. Chaque échantillon d'ADN a été amplifié deux fois lors d'une même PCR, lesquels ont été déposés côte à côte sur le gel pour contrôler la reproductibilité et faciliter l'analyse.

\subsection{Analyse des données}

La présence des fragments a été déterminée visuellement et introduite dans une matrice de données binaire $0 / 1$.

Comme les analyses de variance moléculaire (AMOVA [49]) sont limitées à trois niveaux hiérarchiques, plusieurs analyses séparées ont été réalisées. Toutes les AMOVA ont été calculées en utilisant ARLEQUIN vers. 2.000 [49] et 1000 permutations pour les tests de signification. Du point de vue de la différenciation génétique entre les pins 
d'altitude et de plaine, une AMOVA a été calculée avec les douze populations groupées en six paires. Par la suite, la matrice des valeurs de différenciation génétique $\Phi_{\mathrm{ST}}$, qui sont analogues aux valeurs $\mathrm{F}_{\mathrm{ST}}$, a été calculée pour les douze populations.

Dans le but de mettre en évidence d'éventuelles corrélations entre les marqueurs génétiques et la vitalité, une AMOVA a été calculée en groupant les individus à forte vitalité et à faible vitalité de chaque population en deux sous-populations, pour chacune des six paires de populations. Pour étudier la relation entre la diversité génétique d'une population et son état de santé, la variance moléculaire (obtenue par une AMOVA, SS $/(n-1)$; SS = somme des distances quadratiques, $n=$ nombre d'individus) de chaque population a été calculée (selon [14]) avec WinAmova vers. 1.55 [13]. Les différences entre les variances moléculaires des populations d'altitude (saines) et celles des populations de plaine (dépérissantes) ont été testées (test $t$ ) dans le but de mettre en évidence un possible effet de dérive dû au dépérissement.

\subsection{Analyse de la croissance et de la quantité d'aubier}

La largeur des cernes, le diamètre du cœur et l'âge ont été mesurés pour chacune des deux carottes par arbre à l'aide de la table linéaire Lintab (Rinn S.A.). Les valeurs par individu furent définies par la moyenne des deux mesures. Seules les quatre paires de populations du Valais et celle des Grisons ont été mesurées.

À partir des valeurs annuelles d'accroissement par individu, la courbe des accroissements annuels moyens a été calculée pour chaque population en considérant les données de tous les individus disponibles pour un âge donné. La courbe a été interrompue à l'âge où la valeur provient de la moyenne de moins de trois individus. La courbe de l'accroissement de la surface terrière a été déterminée à partir de cette dernière. Afin de définir la pente des courbes d'accroissement de la surface terrière entre les populations de plaine et d'altitude, une analyse de régression linéaire a été effectuée pour chaque population (Genstat 5, vers. 3.2, Lawes Agricultural Trust, Rothamsted), sur la période allant du $21^{\mathrm{e}}$ cerne à l'âge maximal de la population de plaine respective de chaque paire. La linéarité de l'accroissement en phase juvénile ( $<21$ ans à $1,3 \mathrm{~m}$ de hauteur) n'a en effet pas pu être admise. La signification des différences de pente entre les droites de régression linéaire a ensuite été testée d'après Sokal et Rohlf [52]. La distribution des résidus n'a pas indiqué qu'une des hypothèses ayant trait à la distribution normale des données ait été violée.

La différence entre le nombre de cernes d'aubier des individus de plaine et de ceux d'altitude a été testée à l'aide d'une analyse de variance (general linear model, randomized block design; SyStat, vers. 10, SPSS Inc.).

\section{RÉSULTATS}

Les amplifications RAPD avec les quatre amorces choisies ont donné 38 bandes reproductibles et fiables pour l'interprétation, d'une longueur de 400 à 1380 paires de bases (Tab. II). Malgré huit marqueurs monomorphes, tous les individus ont pu être distingués à l'aide des 30 fragments polymorphes, ce qui confirme le pouvoir de résolution attribué aux marqueurs RAPD [40]. Les fréquences des marqueurs polymorphes varient de 0,23 à 0,99 .
Tableau III. Différenciation génétique $\left(\Phi_{\mathrm{ST}}\right.$, moitié inférieure de la matrice) et seuils de signification (moitié supérieure de la matrice) entre six paires de populations de Pinus sylvestris.

\begin{tabular}{ccccccc}
\hline & $\mathrm{St}$ & $\mathrm{Br}$ & $\mathrm{Sa}$ & $\mathrm{Le}$ & $\mathrm{Fi}$ & $\mathrm{Ao}$ \\
\hline $\mathrm{St}$ & & $\mathrm{ns}$ & $\mathrm{ns}$ & $\mathrm{ns}$ & $* * *$ & $\mathrm{~ns}$ \\
$\mathrm{Br}$ & $-0,008$ & & $\mathrm{~ns}$ & $\mathrm{~ns}$ & $*$ & $\mathrm{~ns}$ \\
$\mathrm{Sa}$ & 0,007 & 0,006 & & $* *$ & $* * *$ & $\mathrm{~ns}$ \\
$\mathrm{Le}$ & 0,013 & $-0,003$ & 0,023 & & $*$ & $\mathrm{~ns}$ \\
$\mathrm{Fi}$ & 0,048 & 0,017 & 0,035 & 0,020 & & $* * *$ \\
$\mathrm{Ao}$ & 0,001 & $-0,004$ & $-0,002$ & 0,010 & 0,041 & \\
\hline
\end{tabular}

$* p<0,05$;** $p<0,01$; *** $p<0,001$; ns = non significatif. Les abréviations se réfèrent au tableau I.

Tableau IV. Différenciation génétique entre six paires de populations et entre les deux populations d'une même paire (altitude) de Pinus sylvestris.

\begin{tabular}{lcccc}
\hline Source de variation & SS & d.d.l. & $\%$ de variation & $p$ \\
\hline Paires $\left(\Phi_{\mathrm{CT}}\right)$ & 32,8 & 5 & $-0,76$ & $\mathrm{~ns}$ \\
Altitudes $\left(\Phi_{\mathrm{SC}}\right)$ & 47,3 & 6 & 4,34 & $* * *$ \\
À l'intérieur des populations & 945,9 & 192 & 96,43 & \\
\hline
\end{tabular}

*** $p<0,001 ; \mathrm{ns}=$ non significatif. Les abréviations se réfèrent au tableau I.

Tableau V. Différences orographiques et différenciation génétique $\left(\Phi_{\mathrm{ST}}\right)$ entre des populations de plaine et d'altitude de Pinus sylvestris, pour six paires de populations.

\begin{tabular}{lcccc}
\hline & \multicolumn{2}{c}{ Différences } & \multicolumn{2}{c}{ Différentiation génétique } \\
\cline { 2 - 5 } Paire & Altitude & Exposition & $\Phi_{\mathrm{ST}}$ & $p$ \\
\hline Stalden & $950 \mathrm{~m}$ & Aucune & 0,058 & $* *$ \\
Brigue & $580 \mathrm{~m}$ & N et S & 0,007 & $\mathrm{~ns}$ \\
Salquenen & $810 \mathrm{~m}$ & Aucune & 0,054 & $* *$ \\
Leytron & $650 \mathrm{~m}$ & S et SE & 0,055 & $* *$ \\
Filisur & $600 \mathrm{~m}$ & Aucune & 0,044 & $*$ \\
Aoste & $640 \mathrm{~m}$ & SW et S & 0,042 & $* *$ \\
\hline
\end{tabular}

$* p<0,05 ; * * p<0,01 ; \mathrm{ns}=$ non significatif.

Les valeurs de différenciation génétique entre les six paires ont mis en évidence la paire de Filisur. Cette dernière se distingue de manière significative de chacune des cinq autres paires de populations (Tab. III). Le groupe des populations de plaine et le groupe des populations d'altitude ont présenté une différence significative lors de l'analyse de la variance moléculaire ( $\Phi_{\mathrm{SC}}=4,34 \%$; Tab. IV). La variation génétique située à l'intérieur des populations s'est alors élevée à 96,4\%. Dans les comparaisons individuelles de chaque paire de populations, toutes les populations de plaine et d'altitude d'une même paire ont présenté une différence génétique significative, à l'exception de la paire de Brigue ( $\Phi_{\mathrm{ST}}$; Tab. V).

Les analyses AMOVA des quatre paires de populations situées en Valais n'ont montré aucune différence génétique significative entre les sous-populations composées d'individus présentant un degré de perte d'aiguilles inférieur à $25 \%$ et celles composées d'individus présentant plus de $75 \%$ de perte d'aiguilles $(p>0,05)$. Les populations d'altitude, non touchées par le dépérissement du pin, n'ont pas non plus montré 

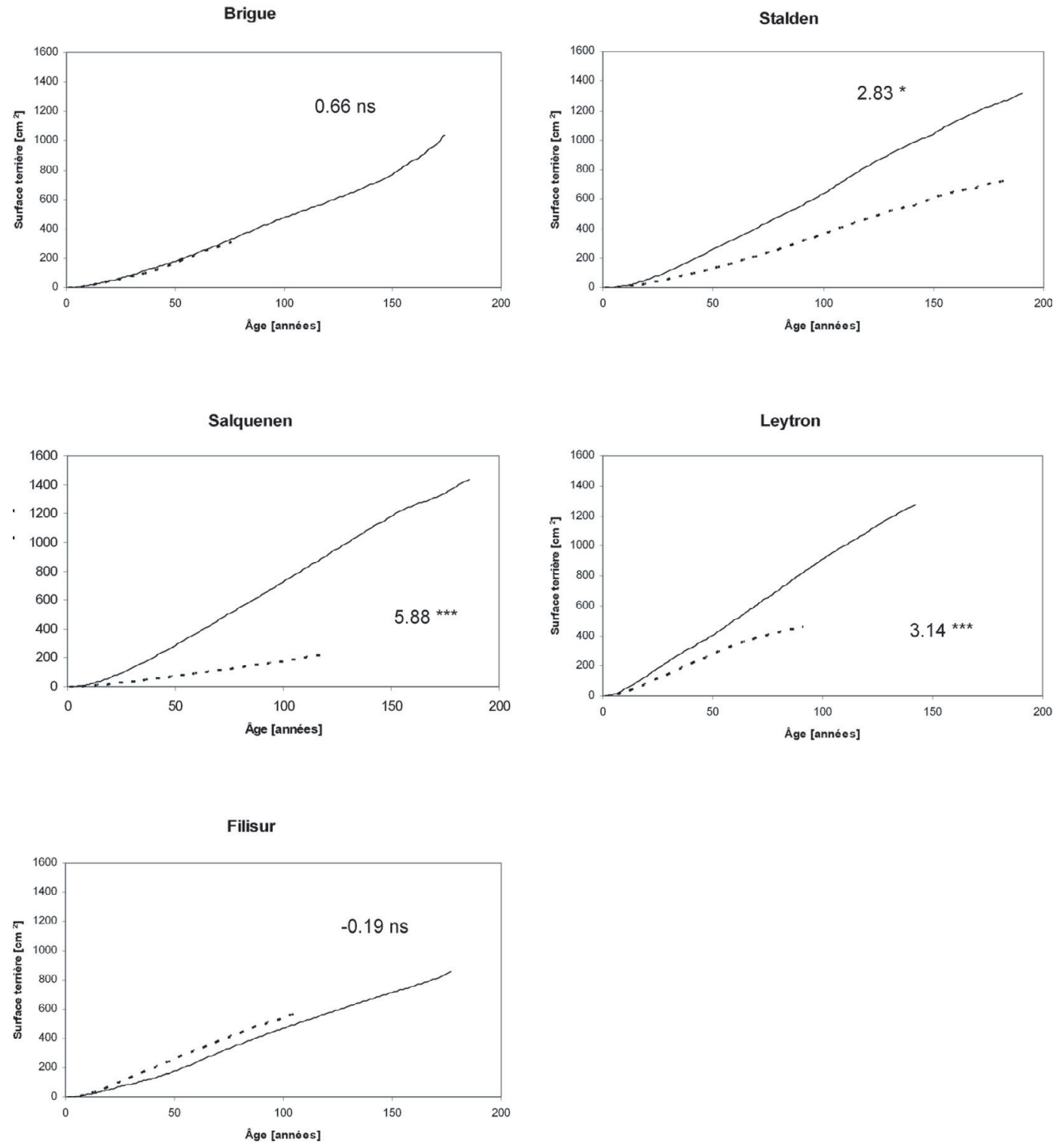

Figure 2. Courbes d'accroissement de la surface terrière, en fonction de l'âge, pour cinq populations de plaine (ligne pointillée) et d'altitude (ligne continue) de Pinus sylvestris. La valeur absolue des différences de pente, selon Sokal et Rohlf [52] et les différences significatives sont mentionnées pour chaque graphique. ${ }^{*} p<0,05 ; * * * p<0,001 ;$ ns $=$ non significatif.

des valeurs de variance moléculaire (Tab. I) plus élevées que celles de plaine $(t=0,848$, d.d.l. $=5, p>0,05)$.

Les populations de plaine sont toutes plus jeunes que les populations d'altitude respectives. Les courbes d'accroissement de la surface terrière des populations de plaine et d'altitude ne se distinguent pas pour toutes les paires de populations (Fig. 2). Les différences de pente sont significatives pour les paires de Stalden $(p<0,05)$, Leytron et Salquenen $(p<0,001)$. Par contre, les pentes des populations de plaine et d'altitude ne se distinguent pas significativement pour Brigue et Filisur.

Le nombre de cernes de bois d'aubier à hauteur de poitrine pour les cinq populations d'altitude et les cinq populations de plaine augmente avec l'âge (Fig. 3). Pour un âge donné, le pin de plaine possède d'autre part un nombre de cernes d'aubier significativement plus élevé que le pin d'altitude (Tab. VI).

\section{DISCUSSION}

Bien que les populations de plaine et d'altitude de chaque paire soient très proches géographiquement, les deux groupes ont présenté une différentiation génétique générale. De même, cinq des six paires analysées ont présenté une différence génétique significative (Tabs. IV et V). L'absence de différence entre les sous-populations à forte vitalité et celles à faible vitalité diminue les sources d'hétérogénéité à l'intérieur des populations échantillonnées, ce qui soutient la validité des analyses en fonction de l'altitude. 


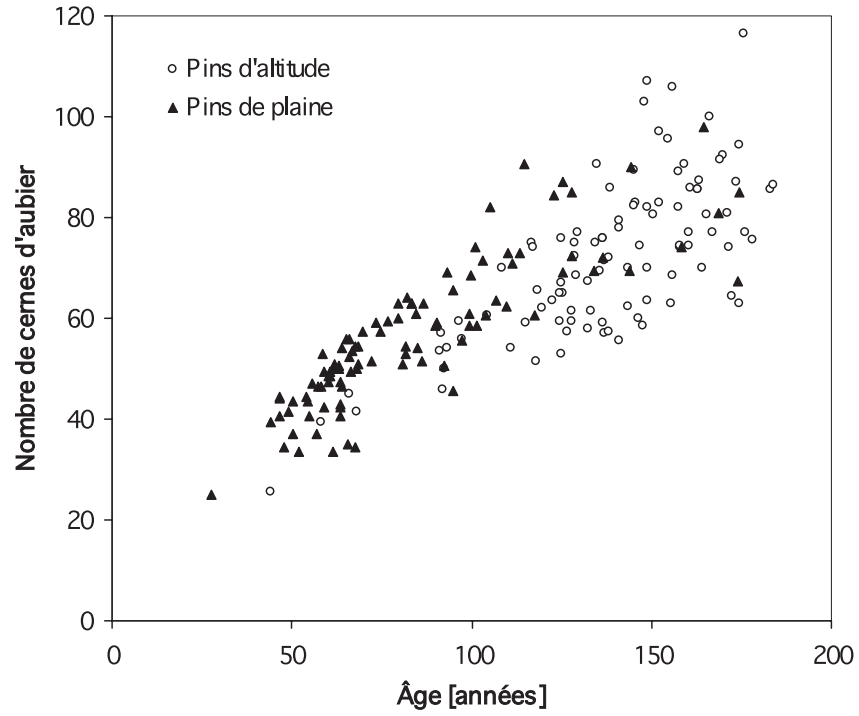

Figure 3. Nombre de cernes de bois d' aubier et âge pour les individus de dix populations de Pinus sylvestris de plaine et d'altitude.

Tableau VI. Analyse de variance du nombre de cernes de bois d'aubier relativement à l'âge entre dix populations d'altitude et de plaine de Pinus sylvestris en Suisse.

\begin{tabular}{lccccc}
\hline Source de variation & SS & d.d.l. & MS & Valeur de F & $p$ \\
\hline Paires & 0,364 & 4 & 0,091 & 10,21 & $<0,001$ \\
Altitudes & 1,587 & 1 & 1,587 & 178,14 & $<0,001$ \\
Erreur & 1,710 & 192 & 0,009 & & \\
\hline
\end{tabular}

\subsection{Différenciation génétique en fonction de l'altitude}

L'ampleur de la différenciation génétique observée entre les populations de plaine et d'altitude en général et à l'intérieur d'une même paire est faible (Tabs. IV et V). Elle dépasse cependant la différenciation de 2 à $3 \%$ mesurée habituellement entre des populations de pin sylvestre d'une même région géographique [e.g. 26]. Les paramètres génétiques des populations estimés avec des RAPD sont en général plus élevés qu'avec d'autres marqueurs. Le fait que seuls les phénotypes et non les génotypes soient considérés dans notre analyse statistique permet pourtant de réduire ce biais [24].

La différenciation génétique observée pourrait provenir de l'adaptation divergente du pin sylvestre en raison d'un facteur de sélection différencié avec l'altitude. Le stress hydrique atteint en effet une ampleur extrême dans la plaine du Rhône [5], la région de Stalden présentant la somme des précipitations la plus faible de Suisse (Ackersand, altitude $700 \mathrm{~m}$, précipitations annuelles $512 \mathrm{~mm}$, [28]). L' augmentation graduelle des précipitations (e.g. Brig, altitude $671 \mathrm{~m}$, précipitations annuelles $717 \mathrm{~mm}$; Simplon village, altitude $1495 \mathrm{~m}$, précipitations $1242 \mathrm{~mm}$ [28]) et la diminution de la température avec l'altitude définissent un gradient hydrique marqué. La différenciation génétique entre le pin d'altitude et le pin de plaine pourrait d'autre part s'expliquer par une origine différente des populations lors de la recolonisation postglaciaire. La comparaison des trois régions étudiées dans cette étude a notam- ment mis en évidence la région des Grisons qui se distingue très significativement de celles du Valais et du Val d'Aoste (Tab. III). L'absence d'un flux génique régional élevé aurait alors été nécessaire pour conserver cette différence jusqu'à ce jour. Compte tenu de la capacité d'expansion des espèces pionnières, évaluée à $1500 \mathrm{~m}$ par an [22], une population provenant d'un refuge de l'Est (Balkans, Carpates) aurait pu atteindre le Nord des Alpes par le plateau suisse avant les populations du refuge des Apennins. Ces populations jouxtant les langues glaciaires auraient ensuite été avantagées pour recoloniser le fond des vallées. Le modèle observé pour le chêne soutiendrait la plausibilité de cette hypothèse [32, 41].

Par contre, l'hybridation introgressive entre le pin sylvestre $(P$. sylvestris) et le pin de montagne ( $P$. mugo ssp. uncinata DC.) joue probablement un rôle marginal dans le processus de différenciation. Bien que, sur le plan morphologique, différentes études indiquent un continuum entre les deux taxons [39], une analyse génétique de populations suisses suspectées intermédiaires a montré que la fréquence des hybrides est en réalité très faible [38].

L'absence de différenciation significative entre les populations de Brigue laisse envisager un modèle de différenciation plus complexe que la simple existence d'une race d'altitude et d'une race de plaine (cf. [19]). Les particularités topographiques de la paire de Brigue et la comparaison des valeurs de $\Phi_{\text {ST }}($ Tab. V) permettent en effet de formuler certaines hypothèses quant aux facteurs de la différenciation observée.

$\mathrm{Au}$ processus de la différenciation génétique s'oppose généralement la force homogénéisante du flux génique [37]. La balance entre le flux génique et la dérive génétique détermine le degré de différenciation et le taux de convergence vers cet équilibre [33]. Chez le pin sylvestre, les conditions de vent, les variations temporelles de la floraison et la durée de réceptivité des fleurs femelles influencent fortement le taux de fécondation croisée [35]. Comme le début de la floraison chez le pin sylvestre dépend de la somme totale des températures et, en raison de la faible durée de réceptivité des fleurs femelles [27], les différences de température avec l'élévation conduisent rapidement à un isolement phénologique des populations [46]. La dispersion des semences ne peut compenser que partiellement cette tendance car la grande majorité des semences de conifères dispersées par le vent atteint le sol dans les premières centaines de mètres [35]. Dans les Carpates, un isolement phénologique presque total a été mesuré entre des populations séparées par une différence d'élévation de 400 m [40]. À Brigue, l'exposition nord du peuplement de plaine opposée à l'exposition sud du peuplement d'altitude (Tab. V) diminue nécessairement les différences écologiques entre les deux stations. Une différence de chaleur insuffisante pour créer la barrière phénologique discutée plus haut pourrait ainsi expliquer l'absence de différenciation génétique entre ces deux populations.

Les vents dominants semblent également jouer un rôle important dans le processus de différenciation observé, parallèlement à la phénologie. Les populations valaisannes de plaine de Salquenen, Leytron et Stalden ne présentent aucune différence génétique significative. Par contre, les trois populations d'altitude correspondantes se distinguent toutes significativement les unes des autres (observations tirées de la matrice des 
valeurs $\Phi_{\mathrm{ST}}$; données non présentées). À l'exemple du régime typique des brises, le relief du Valais induit une canalisation des vents qui suivent plus ou moins fidèlement le modelé du bassin principal et des vallées secondaires [7]. Une forte différenciation génétique entre les peuplements de la plaine du Rhône, balayée quotidiennement par les vents, aurait été inattendue. Par contre, sur les coteaux, les flux géniques pourraient être fortement limités entre des vallées adjacentes. Ceci expliquerait une tendance à la différenciation génétique plus élevée chez les populations d'altitude.

\subsection{Classes de vitalité}

L'absence de corrélation entre les classes de vitalité et les marqueurs génétiques n'exclut pas que la vitalité soit liée aux génotypes. En effet, les RAPD ne sont pas nécessairement liés à des caractères morphologiques, physiologiques ou adaptatifs [20]. Ils couvrent d'autre part une portion du génome relativement réduite. La probabilité d'obtenir un marqueur contenant ou couplé à un gène induisant une résistance ou une différence de vitalité est ainsi très faible. Bien que le taux de défoliation représente un bon indicateur du taux de mortalité annuel [9], l'analyse génétique d'arbres morts aurait peut-être apporté des résultats plus probants. La différenciation spécifique aux marqueurs entre les classes de vitalité s'est avérée très réduite puisqu' un seul des 38 marqueurs a obtenu $12 \%$ de sa variation attribuée aux classes de vitalité.

Les premiers résultats de l'étude du dépérissement des pinèdes en Valais indiquent que l'affaiblissement des individus découle d'une association complexe de facteurs de stress comme la sécheresse [2, 10], le gui (Viscum album ssp. austriacum) [11] et la concurrence [43, 61] tandis que la mort résulte de l'action combinée de plusieurs agents pathogènes secondaires [43]. Plusieurs hypothèses visant à expliquer la répartition spatiale des individus morts sont étudiées actuellement, comme la concurrence, la répartition stochastique des vecteurs de maladie ou l'influence des micro stations. Néanmoins, il demeure légitime d'envisager que le dépérissement diffus observé communément [54] chez le pin sylvestre résulte de caractères inhérents à son écologie génétique. Par exemple, la plasticité permet aux individus de survivre dans des milieux qui ne correspondent pas à leur optimum physiologique. Des modèles développés récemment indiquent en effet qu'un type plastique peut se maintenir dans un environnement suffisamment hétérogène ou en présence d'un flux migratoire élevé, malgré un degré de fitness inférieur aux génotypes spécialisés [56]. L'existence d'un gradient de classes de vitalité dans les populations de pin sylvestre pourrait donner une explication au comportement très hétérogène du pin sylvestre face aux stress.

\subsection{Différences morphologiques avec l'altitude}

La force du transport de l'eau dans le tronc est déterminée par la différence de potentiel hydrique entre les racines et les feuilles. Celle-ci dépend notamment de la hauteur de l'arbre, de l'humidité du sol et de l'air, de la conductivité hydraulique de l'aubier et du comportement des stomates. Lors de différences de potentiel trop élevées, l'effet de cavitation (embolie) induit des conséquences souvent irréversibles pour la plante. La « limitation hydraulique » [45] considère que l'arbre optimise les paramètres de la photosynthèse en fonction du régime hydrique. Cette théorie pourrait expliquer l'accroissement réduit des populations de plaine de Salquenen, Leytron et Stalden et les faibles hauteurs moyennes des peuplements de plaine (Tab. I). Dans la plaine du Rhône valaisanne, le stress hydrique atteint en effet un niveau extrême sur la rive droite du Rhône [5]. Il est intéressant de constater qu'à Brigue, où aucune différence génétique significative n'a été observée, les deux populations ne se distinguent pas non plus par leur croissance en diamètre. À Filisur, on peut d'autre part supposer que le gradient hydrique n'est pas aussi marqué qu'en Valais en raison de l'altitude plus élevée de la population de plaine et d'une continentalité moins marquée (Tiefencastel, fond de vallée, altitude : $822 \mathrm{~m}$, précipitations : $806 \mathrm{~mm}$ [16]).

Le nombre de cernes d'aubier plus élevé chez le pin de plaine que chez le pin d'altitude laisse supposer des équilibres différents pour l'approvisionnement en eau. Dans ces peuplements pas ou très peu exploités, l'accroissement de la surface terrière est linéaire à partir du $20^{\mathrm{e}}$ cerne, ce qui signifie que la surface de chaque nouvelle cerne est constante. Le nombre plus important de cernes de bois d'aubier chez le pin de plaine indique un rapport LA/SA (leaf area/sapwood area) plus élevé que chez le pin d'altitude au même âge. Cette tendance caractérise généralement les individus des milieux xériques [34]. Le changement d'allocation de la biomasse du feuillage vers l'aubier permet en effet de subvenir aux besoins en eau de transpiration plus élevés dans les environnements xériques [8]. Le nombre de cernes d'aubier plus élevé explique finalement le début tardif de la formation du bois de cœur observé chez le pin de plaine [21].

On peut se demander dans quelle mesure les différences morphologiques entre les peuplements proviennent d'un effet de la plasticité individuelle du pin sylvestre [17]. La plasticité et l'adaptation génétique sont souvent considérées comme deux facteurs complémentaires d'adaptation, mais aucune relation univoque entre ces deux variables n'a été trouvée à ce jour [3]. D'après les modèles de Sultan et Spencer [56], l'adaptation génétique pourrait être limitée aux conditions extrêmes, par exemple aux frontières de l'écogramme de l'espèce. Une étude effectuée au sujet de deux types de pin ponderosa de montagne et de milieux xériques suggère à partir d'essais de plantation que de nombreux caractères phénotypiques résultent uniquement de l'effet de la plasticité [30]. Pour notre cas, un essai de provenance serait également nécessaire pour préciser si le pin de plaine et le pin d'altitude correspondent effectivement à des écotypes.

Remerciements : Nos remerciements vont à Markus Van der Meer pour la mesure des carottes, Annie Diarra pour les essais préliminaires de laboratoire, Jean-Philippe Schütz, Rolf Holderegger, Peter Rotach et un lecteur anonyme pour la critique du manuscrit. Notre reconnaissance va également aux Services forestiers de l'État du Valais, des Grisons et du Val d'Aoste qui ont autorisé l'échantillonnage dans leurs peuplements. Nous tenons encore à remercier 
le programme «Dynamique de la forêt» du WSL, représenté par Thomas Wohlgemuth, pour son soutien financier.

\section{RÉFÉRENCES}

[1] Benett K.D., Tzedakis P.C., Willis K.J., Quaternary refugia of north European trees, J. Biogeogr. 18 (1991) 103-115.

[2] Bigler C., Bräker O.U., Bugmann H., Dobbertin M., Rigling A. Drought as inciting mortality factor in Scots pine stands of the Valais, Switzerland, Ecosystems 9 (2006) DOI : 10.1007/s100021005-0126-2 (in press).

[3] Booy G., Hendriks R.J.J., Smulders M.J.M., Van Groenendael J.M., Vosman B., Genetic diversity and the survival of populations, Plant Biol. 2 (2000) 379-395.

[4] Brändli U.-B., Die häufigsten Waldbäume der Schweiz, Ber. Eidg. Forsch. Anst. Wald Schnee Landsch. 342 (1996) 1-279.

[5] Braun-Blanquet J., Die inneralpine Trockenvegetation, Fischer Verlag, Stuttgart, 1961.

[6] Cech T., Tomiczek C., Zum Kiefernsterben in Niederösterreich, Forstschutz-aktuell 17 (1996) 12-13.

[7] De Buman-Ruffieux A.-M., Régimes de vents et qualité de l'air en Valais, Dissertation, Institut de Géographie, Université de Fribourg, Fribourg, 1994.

[8] DeLucia E.H., Maherali H., Carey E.V., Climate-driven changes in biomass allocation in pines, Global Change Biol. 6 (2000) 587-593.

[9] Dobbertin M., Brang P., Crown defoliation improves tree mortality models, For. Ecol. Manage. 141 (2001) 271-284.

[10] Dobbertin M., Hilker N., Rebetez M., Zimmermann N.E., Wohlgemuth T., Rigling A., The upward shift in altitude of pine mistletoe (Viscum album ssp. austriacum) in Switzerland - the result of climate warming? J. Biometeorol. 50 (2005) 40-47.

[11] Dobbertin, M., Mayer, P., Wohlgemuth, T., Feldmeyer-Christe, E., Graf, U., Zimmermann N., Rigling A., The decline of Pinus sylvestris $\mathrm{L}$. forests in the Swiss Rhone Valley - a result of drought stress? Phyton 45 (2005) 153-156.

[12] Dvornyk V., Genetic variability and differentiation of geographically marginal Scots pine populations from Ukraine, Silvae Genet. 50 (2000) 64-69.

[13] Excoffier L., WinAmova vers. 1.55, Analysis of Molecular Variance, Genetics and Biometry Laboratory, University of Geneva, Geneva, 1993

[14] Fischer M., Matthies D., RAPD variation in relation to population size and plant performance in the rare Gentianella germanica (Gentianaceae), Amer. J. Bot. 85 (1998) 811-819.

[15] García-Gil M.R., Mikkonen M., Savolainen O., Nucleotide diversity at two phytochrome loci along a latitudinal cline in Pinus sylvestris, Mol. Ecol. 12 (2003) 1195-1206.

[16] Gensler G.A., Das Klima von Graubünden, Arbeitsberichte der Schweizerischen Meteorologischen Zentralanstalt 77 (1978) 1-122.

[17] Giertych M., Provenance variation in growth and phenology, in: Giertych M., Mátyás C., Genetics of Scots pine, Elsevier, Amsterdam, 1991, pp. 87-101.

[18] Goncharenko G.G., Silin A.E., Padutov V.E., Allozyme variation in natural populations of Eurasian pines, Silvae Genet. 43 (1994) 119-132.

[19] Gugerli F., Eichenberger K. Schneller, J.J., Promiscuity in populations of the cushion plant Saxifraga oppositifolia in the Swiss Alps as inferred from random amplified polymorphic DNA (RAPD), Mol. Ecol. 8 (1999) 1128-1137.

[20] Hearton H.J., Whitkus R., Gómez-Pompa A., Extreme ecological and phenotypic differences in the tropical tree Chicozapote (Manilkara zapota (L.) P. Royen) are not matched by genetic divergence: a random amplified polymorphic DNA (RAPD) analysis, Mol. Ecol. 8 (1999) 627-632.
[21] Hess E., Die autochthonen Föhrenrassen des Wallis, Schweiz. Z. Forstwes. 93 (1942) 1-14.

[22] Hewitt G.M., Some genetic consequences of ice ages, and their role in divergence and speciation, Biol. J. Linn. Soc. 58 (1996) 247-276.

[23] Huntley B., Birks H.J.B., An Atlas of Past and Present Pollen Maps for Europe, Cambridge University Press, Cambridge, 1983.

[24] Isabel N., Beaulieu J., Theriault P., Bousquet J., Direct evidence for biased gene diversity estimates from dominant random amplified polymorphic DNA (RAPD) fingerprints, Mol. Ecol. 8 (1999) 477483.

[25] Jones H.G., Plants and Microclimate. Cambridge University Press, Cambridge, 1992.

[26] Karhu A., Hurme P., Karjalainen M., Karvonen P., Kärkkäinen K., Neale D., Savolainen O., Do molecular markers reflect patterns of differentiation in adaptive traits of conifers? Theor. Appl. Genet. 93 (1996) 215-221.

[27] Koski V., Generative reproduction and genetic processes in nature, in : Giertych M., Mátyás C., Genetics of Scots pine, Elsevier, Amsterdam, 1991, pp. 59-72.

[28] Lingg W.A., Ökologie der inneralpinen Weisstannenvorkommen (Abies alba Mill.) im Wallis (CH), Mit. Eidg. Forsch. Anst. Wald Schnee Landsch. 62 (1986) 1-466.

[29] Maherali H., DeLucia E.H., Xylem conductivity and vulnerability to cavitation of ponderosa pine growing in contrasting climates, Tree Physiol. 20 (2000) 859-867.

[30] Maherali H., Williams B.L., Paige K.N., DeLucia E.H., Hydraulic differentiation of Ponderosa pine populations along a climate gradient is not associated with ecotypic divergence, Funct. Ecol. 16 (2002) 510-521.

[31] Mátyás C., Climatic adaptation of trees: rediscovering provenance tests, Euphytica 92 (1996) 45-54.

[32] Mátyás G., Sperisen C., Chloroplast DNA polymorphisms provide evidence for postglacial re-colonisation of oaks (Quercus spp.) across the Swiss Alps, Theor. Appl. Genet. 102 (2001) 12-20.

[33] McKay J.K., Latta R.G., Adaptive population divergence: markers, QTL and traits, Trends Ecol. Evol. 17 (2002) 285-291.

[34] Mencuccini M., Grace J., Climate influences the leaf/sapwood area ratio in Scots pine, Tree Physiol. 15 (1995) 1-10.

[35] Mitton J.B., The dynamic mating system of conifers, For. Sci. 42 (1992) 197-216.

[36] Molotkov P.I., Patlaj I.N., Systematic position within the genus Pinus and intraspecific taxonomy, in: Giertych M., Mátyás C., Genetics of Scots pine, Elsevier, Amsterdam, 1991, 31-40.

[37] Namkoong G., Koshy M.P., Aitken S., Selection, in: Young A., Boshier D., Boyle T., Forest Conservation Genetics, Principles and Practice, CABI Publishing, Wallingford, 2002, 101-111.

[38] Neet-Sarqueda C., Genetic differentiation of Pinus sylvestris L. and Pinus mugo aggr. populations in Switzerland, Silvae Genet. 43 (1994) 207-215.

[39] Neet-Sarqueda C., Plumettaz Clot A.-C., Bécholey I., Mise en évidence de l'hybridation introgressive entre Pinus sylvestris L. et Pinus uncinata D.C. en Valais (Suisse) par deux méthodes multivariées, Bot. Helv. 98 (1988) 161-169.

[40] Nybom H., Bartish I.V., Effects of life history traits and sampling strategies on genetic diversity estimates obtained with RAPD markers in plants, Perspect. Plant Ecol. Evol. Syst. 3 (2000) 93-114.

[41] Petit R.J., Csaikl U.M., Bordács S., Burg K., Coart E., Cottrell J., van Dam B., Deans J.D., Dumolin-Lapègue S., Fineschi S., Finkeldey R., Gillies A., Glaz I., Goicoechea P.G., Jensen J.S., König A.O., Lowe A.J., Madsen S.F., Mátyás G., Munro R.C., Olalde M., Pemonge M.-H., Popescu F., Slade D., Tabbener H., Taurichini D., de Vries S.G.M., Ziegenhagen B., Kremer A. Chloroplast DNA variation in European white oaks: phylogeography and patterns of diversity based on data from over 2600 populations, For. Ecol. Manage. 156 (2002) 5-26. 
[42] Rebetez M., Dobbertin M., Climate change may already threaten Scots pine stands in the Swiss Alps, Theor. Appl. Climatol. 79 (2004) 1-9.

[43] Rigling A., Cherubini P., Wieso sterben die Waldföhren im «Telwald» bei Visp? Schweiz. Z. Forstwes. 150 (2002) 113-131.

[44] Rigling A., Weber P., Cherubini P., Dobbertin M., Walddynamische Prozesse und Jahrringe - Bestandesdynamik zentralalpiner Waldföhrenwälder aufgezeigt anhand dendroökologischer Fallstudien aus dem Wallis, Schweiz. Z. Forstwes. 6 (2004) 178-190.

[45] Ryan G.M., Yoder B.J., Hydraulic limits to tree height and tree growth, BioScience 47 (1997) 235-242.

[46] Sannikov S.N., Isolation and types of boundaries between Scots pine populations, Ekologiya 1 (1993) 4-11.

[47] Sannikov S.N., Petrova I.V., Ryabokon S.M., Dukharev V.A., Sannikova N.S., Genetic differentiation of bog and dry-valley populations of Pinus sylvestris in western Siberia, Ekologiya 6 (1989) $39-44$.

[48] Sannikov S.N., Semerikov V.L., Petrova I.V., Filippova T.V., Genetic differentiation of Scots pine populations of the Carpathians and the Russian plain, Ekologiya 3 (1997) 163-167.

[49] Schneider S., Roessli D., Excoffier L., Arlequin vers. 2.000: a software for population genetic data analysis, Genetics and Biometry Laboratory, Department of Ecology and Anthropology, University of Geneva, Geneva, 2000.

[50] Sellin A., Sapwood amount in Picea abies (L.) Karst. determined by tree age and radial growth rate, Holzforschung 50 (1996) 291-296.

[51] Sinclair W.T., Morman J., Ennos R.A., The postglacial history of Scots pine (Pinus sylvestris L.) in western Europe: evidence from mitochondrial DNA variation, Mol. Ecol. 8 (1999) 83-88.
[52] Sokal R., Rohlf F.J., Biometry. The Principles and Practice of Statistics in Biological Research, Freeman, New York, 1995.

[53] Soranzo N., Alía R., Provan J., Powell W., Patterns of variation at a mitochondrial sequence-tagged-site locus provides new insights into the postglacial history of European Pinus sylvestris populations, Mol. Ecol. 9 (2000) 1205-1211.

[54] Stephan B.R., Inheritance of resistance to biotic factors, in: Giertych M., Mátyás C., Genetics of Scots pine, Elsevier, Amsterdam, 1991, pp. 205-217.

[55] Stierlin H.-R., Müller E., Rauber K., Kronenbilder mit Nadel- und Blattverlustprozenten, Sanasilva, Eidgenössische Forschungsanstalt für Wald, Schnee und Landschaft, Birmensdorf, 1986.

[56] Sultan S.E., Spencer H.G., Metapopulation structure favors plasticity over local adaptation, Am. Nat. 160 (2002) 271-283.

[57] Tobolski J.J., Hanover J.W., Genetic variation in the monoterpenes of Scots pine, For. Sci. 17 (1971) 293-299.

[58] Vertui F., Tagliaferro F., Scots Pine (Pinus sylvestris L.) die-back by unknown causes in the Aosta Valley, Italy, Chemosphere 36 (1998) 1061-1065.

[59] Volosyanchuk R.T., Pinus sylvestris L., in: Pines of Silvicultural Importance, CABI, New York, 2002, pp. 449-466.

[60] Waring R.H.P.E., Schroeder P.E., Oren R., Application of the pipe model theory to predict canopy leaf area, Can. J. For. Res. 12 (1982) 556-560.

[61] Weber P., Intra- and interspecific competition in mixed Pinus sylvestris and Quercus pubescens stands - Modelling stand dynamics based on tree-ring analysis, Ph. D. thesis ETH No. 16235, ETH Zürich, Zürich, 2005.

[62] Whitehead D., Edwards W.R.N., Jarvis P.G., Conducting sapwood area, foliage area, and permeability in mature trees of Picea sitchensis and Pinus contorta, Can. J. For. Res. 14 (1984) 940-947. 
